\title{
Hierarchical Ring Network Design Using Branch-and-Price
}

\author{
TOMMY THOMADSEN \\ THOMAS STIDSEN \\ Informatics and Mathematical Modelling, Technical University of Denmark, \\ DK-2800 Kongens Lyngby, Denmark
}

tt@imm.dtu.dk tks@imm.dtu.dk

Received May 21, 2004; Accepted October 19, 2004

\begin{abstract}
We consider the problem of designing hierarchical two layer ring networks. The top layer consists of a federal-ring which establishes connection between a number of node disjoint metro-rings in a bottom layer. The objective is to minimize the costs of links in the network, taking both the fixed link establishment costs and the link capacity costs into account.

Hierarchical ring network design problems combines the following optimization problems: Clustering, hub selection, metro ring design, federal ring design and routing problems. In this paper a branch-and-price algorithm is presented for jointly solving the clustering problem, the metro ring design problem and the routing problem. Computational results are given for networks with up to 36 nodes.
\end{abstract}

Keywords: ring network design, hierarchical network design, branch-and-price

\section{Introduction}

Design of survivable communication networks is important for at least two reasons. First of all there is a growing reliance on electronic communication in society. Secondly failures (e.g. a link failure) may have a large impact, given the high capacity of links.

Self Healing Rings (or rings for short) have been widely used to ensure survivable communication for several reasons. First of all, the rings are pre-configured such that the only nodes that need to do re-routing in case of a link failure are the two endpoint nodes of the failed link. Thus no communication with other nodes is necessary making ring protection fast. Furthermore the node equipment is cheap to build and protection does not require the involvement of an expensive network management system.

Larger networks consist of several interconnected rings, since it is neither possible nor beneficial to restrict the entire network topology to a single ring. One possible way to interconnect the rings is in a hierarchy. Hierarchical networks have existed for decades and were introduced because of the limited switching capabilities in the telephone systems. Hierarchies are still used since they divide the network in sub-networks which can to some extend be treated independently, easing maintenance and upgrade.

In this paper we consider the design of hierarchical ring networks (HRNs), i.e. hierarchical networks where each sub-network is a ring. We assume that communication 


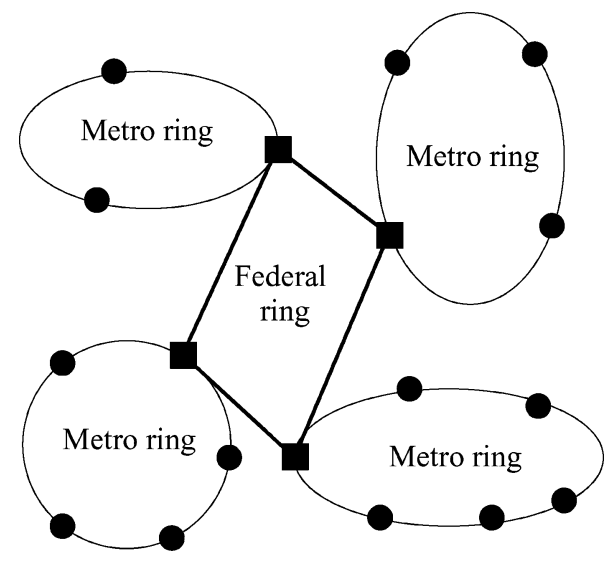

Figure 1. A two layer hierarchical ring network.

demands are given and determine a HRN which satisfies the communication demands as cheaply as possible. We present models and algorithms for two layers only, but both models and algorithms can be generalized to more layers. We denote the ring in the top layer the federal-ring, and the node disjoint rings in the bottom layer, the metro-rings. See figure 1 for an example of a HRN. We consider single homing, i.e. exactly one node from each metro-ring is in the federal-ring. This node is called the hub node.

The HRN design problem belongs to the more general class of hierarchical network design problems which jointly considers hub location and network design. For an excellent survey of this area we refer to [9]. In [9] the hierarchical network design problem is decomposed into a number of smaller optimization problems:

Clustering: Decide which nodes should belong to the same metro-network.

Hub Selection: For each metro-network select hub nodes to connect the metro-networks to the federal-network.

Metro-Network Design: Determine the best network to connect the nodes in each metro-network.

Federal-Network Design: Determine the best federal-network connecting the hub nod-es.

Routing: Route the communication demands, minimizing the capacity usage in nodes and links.

An approach to the hierarchical network design problem is to solve it step by step by solving one or more of the above smaller optimization problems in each step. This is what most papers suggest, including this paper. This means that the hierarchical network design problem is solved by first solving e.g. the clustering problem and the metronetwork design problem, then the hub selection problem and the federal-network design 
problem and finally the routing problem. The optimization process is much simpler but a suboptimal solution of the hierarchical network design problem may be obtained.

The main contribution of the paper is the implementation of a branch-and-price algorithm which can be used to solve to optimality a modified model which includes the clustering problem, the metro-ring design problem and the routing problem. We refer to this problem as the modified HRN problem. The hub selection problem and the federalring design problem can be solved jointly afterwards and is a Generalized Travelling Salesman Problem considered in e.g. [3]. We discuss the modified HRN problem and point out under what circumstances an optimal solution for the modified problem is optimal for the original optimization problem. The problem modification has previously been put forward and used for implementing heuristics but it has not been analysed in detail. Optimal solutions have previously been obtained for networks with up to 12 nodes and used for comparison with heuristic values. Our branch-and-price algorithm can in general solve instances with 20 nodes and for problems with special structure up to 36 nodes.

The outline of the paper is as follows. In Section 2, we discuss related papers. In Section 3 we consider the problem modification of the clustering problem and the metro-ring design problem. In Section 4, the integer linear formulation of the modified HRN problem is given and in Section 5 we describe how the integer linear model can be solved using a branch-and-price algorithm. In Section 6 we give some computational results and suggest some directions for future research. Finally we give some concluding remarks in Section 7.

\section{Previous work}

Because of the importance of ring protection, a significant amount of work has been carried out regarding the design of ring networks. In this section some related papers are briefly discussed.

In [1] a two layer HRN design problem is studied. The hubs are assumed given, thus eliminating the hub selection problem and reducing the federal-network design problem to a Travelling Salesman Problem. The focus is on the clustering problem and the metronetwork design problem. For the optimization of the metro-network design problem, a heuristic with guaranteed worst case performance is suggested. By constructing the rings, the clustering problem is implicitly solved. Finally, the routing problem is ignored, i.e. the capacity costs of the network is not considered.

In [6] a more real-world version of the HRN design problem is studied. Besides requiring some metro-networks to be rings, some metro-networks are allowed to be treelike. This is achieved by dividing nodes into two groups, some which require ring protection and some which do not. The communication demand pattern assumes that each demand node only requires to communicate with one or a few service nodes. This assumption simplifies the routing problem. The problem is solved in steps using heuristics to first determine the clusters, select hubs, design the metronetworks and design the federal-network. For each problem tailored heuristics are 
used, which makes the combined optimization algorithm fast and applicable to what-if analyses.

In [8] another type of hierarchical network design problem is considered. Each sub-network is allowed to consist of several rings, which are connected to the same hub nodes. Again the problem is solved in steps. First the clusters are determined and the hubs selected, using the column generation method from [11]. The objective is to maximize the traffic within the metro-networks. Secondly the metro-network design problem is solved for each of the clusters separately by a column generation algorithm. The algorithm is used to minimize the cost of the rings which connects all the nodes in each of the metro-networks to the hubs. The costs includes both link costs and node costs. The federal-network design problem and the routing problem is not considered. Finally the developed algorithm is tested on a real world telecommunication network from Korea Telecom.

Another type of the hierarchical network design problem is suggested in [7]. The focus in this article is the clustering problem. The nodes are clustered in order to minimize the number of necessary clusters, i.e. metro-networks, constrained by the capacity of the metro-rings and the federal-ring. The cost of the metro-ring structures and the federalring structures are thus not considered.

The hardness of the routing problem in combination with one or more other problems is illustrated in the paper [5]. Here the routing costs are considered in combination with the logical design of the rings, i.e. which nodes in the rings should contain add-drop equipment. An integer linear program is presented, but as it is pointed out, it is clearly not suitable to exact solution approaches. Instead a tabu search heuristic for the combined problem is presented. The main problem is that given a complete ring-network design, the routing problem is itself a multicommodity optimization problem. Hence, for each iteration in the tabu-search heuristic, many such multicommodity optimization problems needs to be solved. The algorithm is tested on real world examples with up to 48 nodes.

In [14] the modified HRN problem was introduced and this was further developed in $[15,16,17,18]$. We use the same idea of a modified HRN problem in this paper, and the problem modification is described in greater detail in Section 3. In the papers $[14,15,16,17,18]$ both a heuristic and an enumerative scheme is described, but the number of possible networks grows exponentially, making the enumerative scheme useless except for small and trivial instances (less than 10 nodes). The heuristic on the other hand is able to handle large networks, but gives no guarantee regarding the quality of the solutions obtained.

In [12] an integer linear program of the modified HRN problem is presented. Optimal solutions can in some cases be obtained using the model, for networks with up to 12 nodes and a maximum of 4 nodes in the metro-rings. The focus of the paper is a "partition, construct and perturb" heuristic. This heuristic is compared with optimal solutions when these can be obtained and with the heuristics from [14]. It is concluded that better results than Shi and Fonseka are in general obtained. 


\section{The modified HRN problem}

Let the network $G(V, E)$ where $V$ is the set of nodes and $E$ is the set of possible bidirectional links. Let $D$ be the set of demands, let $R^{\text {met }}$ be the set of possible metrorings and $R^{\text {fed }}$ the set of possible federal-rings. For $r \in R^{\text {fed }}$ or $r \in R^{\text {met }}, r \subseteq E$, i.e. $r$ is a subset of links, and the links induce a ring. Let $d_{i j}^{\prime}, i j \in D$ denote the demand for communication flow between node $i \in V$ and $j \in V$. Also let $c_{e}$ be the fixed cost for establishing link $e$ and correspondingly let the cost per capacity unit on link $e$ be $b_{e}$.

The purpose of modifying the problem is to obtain a formulation which includes the cluster problem, the metro-network design problem and implicitly includes the routing problem. Thus no routing variables are necessary. This is possible, since we consider unidirectional self-healing rings. The modification also allows a decomposition of the total cost into costs for each ring which can be measured independently.

The cost of a HRN is assumed to depend solely on the links used by the rings in the network and the capacity of these links, i.e. the fixed cost and the capacity cost respectively. Thus the cost of a HRN is as given in equation (1), where $r^{\text {fed }} \in R^{\text {fed }}$ is the federal-ring, $\bar{R}^{\text {met }} \subset R^{\text {met }}$ is the set of node disjoint metro-rings covering all nodes and finally $C A P_{r}$ is the minimal capacity required on each link of ring $r$ to service the traffic flow.

$$
\sum_{e \in \mathrm{r}^{\mathrm{fed}}} c_{e}+C A P_{r_{\mathrm{red}}} \cdot \sum_{e \in r^{\mathrm{fed}}} b_{e}+\sum_{r \in \bar{R}^{\mathrm{met}}}\left(\sum_{e \in r} c_{e}+C A P_{r} \cdot \sum_{e \in r} b_{e}\right)
$$

The fixed cost of the federal-ring is left as a separate optimization problem, i.e. the HRN cost is initially approximated by the fixed cost of the metro-rings and the capacity cost:

$$
\sum_{r \in \bar{R}^{\text {met }}} \sum_{e \in r} c_{e}+C A P_{r^{\mathrm{fed}}} \cdot \sum_{e \in r^{\mathrm{fed}}} b_{e}+\sum_{r \in \bar{R}^{\mathrm{met}}} C A P_{r} \cdot \sum_{e \in r} b_{e}
$$

We consider unidirectional self-healing rings, for which it holds that communication flow in the ring takes up capacity in all links in the ring. Thus if a demand $i j \in D$ traverse a ring, it takes up capacity $d_{i j}^{\prime}$ in all links on the ring. Assume that $B$ is the average capacity cost per ring per unit of demand. An estimate of the capacity cost for satisfying the demand $d_{i j}^{\prime}$ is $B d_{i j}^{\prime}$ if $i$ and $j$ are in the same metro-ring and $3 B d_{i j}^{\prime}$ if $i$ and $j$ are in different metro-rings, since three rings are in that case traversed (two metro-rings and the federal-ring). Also the capacity cost can be expressed as a worst case cost, $K=3 B \sum_{i j \in D} d_{i j}^{\prime}$ corresponding to that all demands traverse three rings minus a savings obtained by handling communication demands within metro-rings. Denote by $D_{r}^{\text {met }} \subset D$ the set of demands handled within metro-ring $r$. In that case the capacity cost can be estimated as follows.

$$
C A P_{r^{\text {fed }}} \cdot \sum_{e \in r^{\text {fed }}} b_{e}+\sum_{r \in \bar{R}^{\text {met }}} C A P_{r} \cdot \sum_{e \in r} b_{e} \approx K-2 B \sum_{r \in \bar{R}^{\text {met }}} \sum_{i j \in D_{r}^{\text {met }}} d_{i j}^{\prime}
$$


The total HRN cost is then estimated by the following.

$$
K+\sum_{r \in \bar{R}^{\text {met }}} \sum_{e \in r} c_{e}-2 B \sum_{r \in \bar{R}^{\text {met }}} \sum_{j \in D_{r}^{\text {met }}} d_{i j}^{\prime}
$$

The intuition behind this rewrite is, that minimizing the capacity cost corresponds to maximizing the communication demand handled within metro-rings. This is in good agreement with previous recommendations [4] and what has been done in e.g. [11]. Note that $2 B$ will have to be experimentally determined. Different values of $2 B$ will result in different cost structures, e.g. a low $2 B$ will correspond to the case where the capacity cost is higher in the federal-ring than in the metro-rings.

The cost per ring per unit of demand may be far from constant (i.e. deviate considerably from $B$ ). However if the capacity cost reflects a cost of node-equipment rather than a cost proportional to the distance between nodes, $B$ is thus proportional to the number of nodes in the rings. In that case it makes much more sense to have a known, fixed $B$ corresponding to a known fixed number of nodes in the rings, and in particular $[14,15,16]$ study such networks. For HRNs where the capacity cost per ring per unit of demand is not $B$ in all cases, optimal solutions for the modified problem may not be optimal in the original problem.

Note that the cost can now be decomposed into costs minus a reward for each metro-ring plus a constant $K$, which can be measured independently. Thus the cost for metro-ring $r \in R^{\text {met }}$ is:

$$
c_{r}=\sum_{e \in r} c_{e}-2 B \sum_{i j \in D_{r}^{\text {met }}} d_{i j}^{\prime}
$$

Consider the demand $d_{i j}^{\prime}$ where $i$ and $j$ are in different metro-rings, $i$ is in the federal-ring and $r$ is the metro-ring including $i$. In that case equation (4) includes a cost for routing $d_{i j}^{\prime}$ in $r$, but $d_{i j}^{\prime}$ need not be routed "from $i$ via $r$ to $i$ "- - there is no need to route it in $r$ at all. Thus additional savings should be included if $i$ is in the federal-ring. This saving is included as a reward on nodes when the federal-ring is designed. The node reward is the sum of all demands starting or ending in the node.

\section{The problems}

Given the modification of the problem, the idea is now to select the lowest cost set of metro-rings, which includes nodes exactly once, i.e. a set-partitioning problem. However, since there are too many metro-rings to pregenerate all, we generate metro-rings when needed. Thus what we describe is actually a column generation algorithm or, since branching is needed to get integer solutions, an integer programming column generation algorithm, also known as branch-and-price [2, 20].

In this section we will describe the two problems we need to solve; the ringpartitioning problem (which is a set-partitioning problem) and the ring-generation problem. We will describe the branch-and-price algorithm in detail in Section 5. 
When the metro-rings have been designed, the federal-ring is designed as the shortest ring, which includes exactly one node from each metro-ring and takes into account node rewards as described in the previous section. This is a Generalized Travelling Salesman Problem which can be solved using a branch-and-cut algorithm as done in [3]. This problem seems to be easier than the ring-generation problem which is solved many times, and thus the design of the federal-ring is not the bottleneck of the algorithm. We will not consider the design of the federal-ring any further in this paper.

\subsection{The ring-partitioning problem}

Given a set of metro-rings $R \subset R^{\text {met }}$, the ring-partitioning problem is the problem of choosing the lowest cost subset of metro-rings in $R$, such that all nodes are covered exactly once. Define $p_{i r}=1$ if node $i$ is part of ring $r, 0$ otherwise. The variables $u_{r}$ is 1 if ring $r$ is selected, 0 otherwise. The ring-partitioning problem is then:

$$
\begin{array}{ll}
\min & \sum_{r \in R} c_{r} \cdot u_{r} \\
\text { s.t. } & \sum_{r \in R} p_{i r} \cdot u_{r}=1 \quad \forall i \in V\left(\pi_{i}\right) \\
& u_{r} \in\{0,1\}
\end{array}
$$

The objective 6 is the total cost of selecting metro-rings, where $c_{r}$ is defined in equation (5). Constraints (7) ensure that each node is in exactly one metro-ring and constraints (8) are the integer domain constraints. Finally $\pi_{i}$ are the dual variables for constraints (7). The problem obtained by relaxing constraint (8) is denoted the relaxed ring-partitioning problem. If branching is necessary, additional constraints are added, see Section 5.1. Rings are iteratively generated and added to $R$. The ring-generation problem is described in the following section.

\subsection{The ring-generation problem}

The objective of the ring-generation problem is based on the cost in equation (5). However this cost does not include any information on which other rings are in $R$, and thus it is possible that a node will never be included in any ring. The idea is to add a reward to the objective, which reflects how difficult a node is to cover in the ring-partitioning problem given the current set of rings $R$. A node is difficult to cover if e.g. a single ring $r \in R$ contains the node and thus $r$ need to be selected regardless of the cost. If a node $i$ is difficult to cover a high reward is put on including $i$ in a ring. The reward used is the value of the dual variables in the optimal solution to the ring-partitioning problem, $\pi_{i}$.

Let $d_{i j}=B \cdot d_{i j}^{\prime}$, let $n(r) \subseteq V$ be the nodes in $r$ and let $D_{r} \subset D$ be the set of demands which start and end in $r$. Formally, we generate the ring with most negative 
reduced cost, where the reduced cost is given by the following equation.

$$
c_{r}-\sum_{i \in n(r)} \pi_{i}=\sum_{e \in r} c_{e}-\sum_{i j \in D_{r}} d_{i j}-\sum_{i \in n(r)} \pi_{i}
$$

We assume an upper limit, $m$ is given on the number of nodes in the ring. Define the following variables, $y_{i}=1$ if node $i$ is in the ring, 0 otherwise, $x_{e}=1$ if link $e$ is in the ring, 0 otherwise and $z_{i j}=1$ if demand $i j$ can be handled by the ring, otherwise 0 . (Equivalently, $z_{i j}=1$ if $y_{i}=1$ and $y_{j}=1$, otherwise 0 .)

For $S \subset V$, let $\delta(S) \subset E$ denote the set of edges with an endpoint in $S$ and an endpoint not in $S$. Then the ring-generation problem can be stated as follows.

$$
\begin{array}{rrr}
\min \sum_{e \in E} c_{e} \cdot x_{e}-\sum_{i j \in D} d_{i j} \cdot z_{i j}-\sum_{i \in V} \pi_{i} \cdot y_{i} \\
\text { s.t. } \sum_{e \in \delta\{(i\})} x_{e}=2 y_{i} & \forall i \in V \\
z_{i j} \leq y_{i} & \forall i j \in D \\
z_{i j} \leq y_{j} & \forall i j \in D \\
z_{i j} \geq y_{i}+y_{j}-1 & \forall i j \in D \\
\sum_{i \in V} y_{i} \leq m & \\
\sum_{e \in \delta(S)} x_{e} \geq 2\left(y_{k}+y_{l}-1\right) & \\
\forall S \subset V, 3 \leq|S| \leq n-3, k \in S, l \notin S \\
x_{e} \in\{0,1\}, y_{i} \in\{0,1\}, z_{i j} \in\{0,1\}
\end{array}
$$

The objective (11) corresponds exactly to the reduced cost given in equation (9). If a node is selected $\left(y_{i}=1\right)$, two links should be incident to node $i$, which is ensured by constraint (11). If both nodes $i$ and $j$ are selected the variable $z_{i j}=1$, which is ensured by the constraints (12), (13) and (14). The number of nodes in the rings is bounded by the hop constraint (15). Subtour elimination constraints (16) ensure that a single ring is generated and finally integer solutions are ensured by the domain constraints (17).

We solve the ring-generation problem by branch-and-cut as described in [19], where the subtour elimination constraints are generated as needed. Also [10] describes cuts which may improve the performance of the branch-and-cut algorithm. The ringgeneration problem is a generalization of the (Selective) Travelling Salesman Problem and of the Quadratic Knapsack problem and thus we denote it the Quadratic Selective Travelling Salesman Problem.

If branching is necessary, additional terms are added to the objective function and additional constraints are added. These additions are described in Section 5.1. 


\section{The branch-and-price algorithm}

The branch-and-price algorithm is described in pseudo code in figure 2. The main idea in a branch-and-price algorithm is to perform the bounding in a branch-and-bound algorithm using column generation. The algorithm maintains an incumbent, i.e. the lowest cost feasible solution known, and a set of branch-nodes, i.e. a set of relaxed ringpartitioning problems. Initially the set of branch-nodes contains the ring-partitioning problem without any branching decisions. A branch-node corresponding to a relaxed ring-partitioning problem is solved using column generation in the inner while loop. It is resolved in each iteration of the inner while loop and a ring is generated by the ring-generation problem. If no ring exists with negative reduced cost the value of the ring-partitioning problem is a lower bound. This lower bound is used in the outer loop which is the branch-and-bound part of the algorithm.

In the outer loop it is checked whether the optimal solution to the relaxed ringpartitioning problem solution is feasible, i.e. integer, or if it is a lower bound only. If the solution is integer and better than the current incumbent, the incumbent is updated and that branch is fathomed. If the solution is fractional, the lower bound is compared with the current incumbent and if it is worse, the branch is fathomed. If neither is the case, branching is performed.

\subsection{Ryan-foster branching}

Branching in a branch-and-price algorithm is more complicated than in a standard branch-and-bound algorithm. We use Ryan-Foster branching [13] to obtain integer

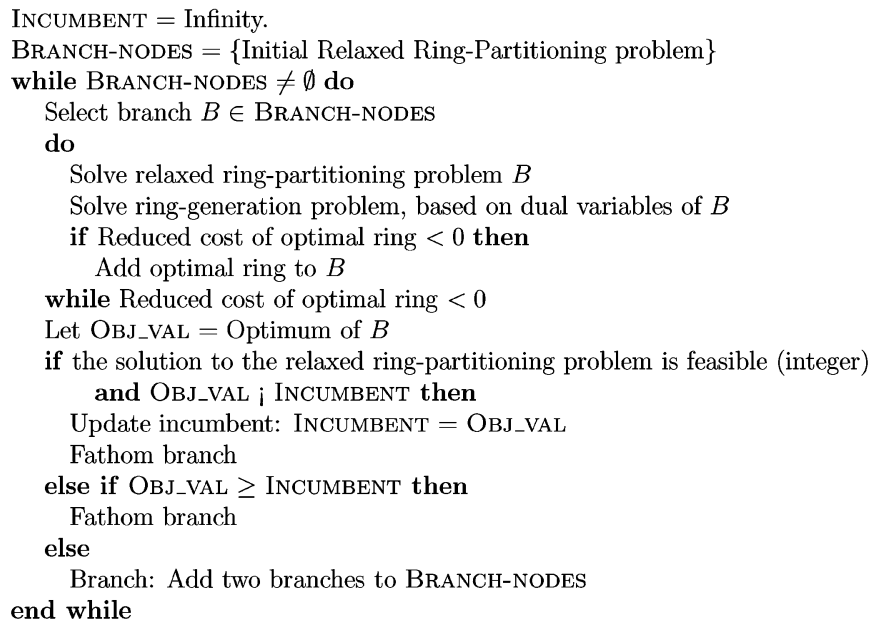

Figure 2. The Branch-and-Price algorithm. 
solutions. This is possible since all coefficients of all constraints in the ring-partitioning problem are 0 or 1 and all right hand sides are 1, see constraint (7).

Consider constraint $i$. Since the right hand side is 1 and variables have to be integer, exactly one ring with $p_{i r}=1$ has to be selected $\left(u_{r}=1\right)$. For all other selected rings, $p_{i r}=0$. We say that "node $i$ is covered by ring $r$ ". The idea is now to identify a set of rings $S \subset R^{\text {met }}$ and create two branches, (1) node $i$ has to be covered by a ring in $S$ and (2) node $i$ has to be covered by a ring not in $S$. The question is now, how do we select $i$ and $S$.

Assume node $i$ is partially covered by more than one ring, and assume ring $r$ is one of these rings (i.e. $0<u_{r}<1$ ). Usual variable branching corresponds to letting $S=\{r\}$, thus the branches will be $u_{r}=1$ and $u_{r}=0$. This sort of branching is not suitable in a column generation algorithm for several reasons all related to the vast amount of variables that exists (but are not explicitly known). First of all since we set $u_{r}=0$ in the ring-partitioning problem, $r$ usually has a negative reduced cost and hence when solving the ring-generation problem, $r$ will be generated again. This can be handled by modifying the ring-generation problem to specifically exclude $r$. However, usually rings similar to $r$ exists and thus these rings will be generated instead. This means that the bound of the $u_{r}=0$ branch will not improve much when branching and we have an unbalanced branch-tree where the depth is considerable.

The idea is to let $S$ contain several rings and in particular include rings which have not yet been generated (i.e. not in $R$ ). Thus in general $S \backslash R \neq \emptyset$. Identify a fractional ring $\left(0<u_{r}<1\right)$ and two nodes $i$ and $j$ with $p_{i r}=1$ and $p_{j r}=1$. If the solution is fractional, such two nodes always exists. Let $S=\left\{r \in R^{\text {met }} \mid p_{i r}=1 \wedge p_{j r}=1\right\}$, that is the rings that cover both $i$ and $j$. The two branches are thus, (1) $i$ and $j$ are covered by the same ring and (2) $i$ and $j$ are covered by different rings.

A branch decision is identified by a node-pair $\{i, j\}$ and whether $i$ and $j$ should be covered by the same ring or not. For a ring-partitioning problem, we have several such branch decisions of both types. Denote by $B^{\mathrm{SAME}} \subset V^{2}$ the set of branching decisions where node-pairs should be covered by the same ring and correspondingly denote by $B^{\text {DIFF }} \subset V^{2}$ the set of branching decisions where node-pairs should be covered by different rings. Then we add the following constraints to the ring-partitioning problem which implement the actual branching.

$$
\begin{aligned}
& \sum_{\left\{r \in R \mid p_{i r}=1 \wedge p_{j r}=1\right\}} u_{r}=1 \quad \forall\{i, j\} \in B^{\mathrm{SAME}} \quad\left(\gamma_{i j}\right) \\
& \sum_{\left\{r \in R \mid p_{i r}=1 \wedge p_{j r}=1\right\}} u_{r}=0 \quad \forall\{i, j\} \in B^{\mathrm{DIFF}} \quad\left(\delta_{i j}\right)
\end{aligned}
$$

We denote the dual variables of the constraints by $\gamma_{\{i, j\}}$ and $\delta_{\{i, j\}}$ as indicated. The constraints added to the ring-partitioning problem affect the calculation of the reduced costs of rings, thus the objective of the ring-generation problem is changed. Note that $p_{i r}=1 \wedge p_{j r}=1$ exactly if $z_{i j}=1$ in the ring-generation problem. Let $\gamma_{\{i, j\}}=0$ if $\{i, j\} \notin B^{\mathrm{SAME}}$ and $\delta_{\{i, j\}}=0$ if $\{i, j\} \notin B^{\mathrm{DIFF}}$, then the objective of the ring-generation 
problem (see equation (10)) becomes:

$$
\sum_{e \in E} c_{e} \cdot x_{e}-\sum_{i j \in D}\left(d_{i j}+\gamma_{\{i, j\}}+\delta_{\{i, j\}}\right) \cdot z_{i j}-\sum_{i \in V} \pi_{i} \cdot y_{i}
$$

When solving the ring-generation problem, it is furthermore necessary to ensure that only rings which fulfill the branching decisions are generated. This is ensured by the following constraints.

$$
\begin{array}{ll}
y_{i}-y_{j}=0 & \forall\{i j\} \in B^{\mathrm{SAME}} \\
y_{i}+y_{j} \leq 1 & \forall\{i j\} \in B^{\mathrm{DIFF}}
\end{array}
$$

Both constraints allows rings where both $y_{i}=0$ and $y_{j}=0$, but constraints (21) ensure that if node $i$ is selected, then so is $j$ and vice versa. On the other hand, constraints (22) ensure that rings generated include at most one of $i$ and $j$.

\section{Computational results}

To test the branch-and-price algorithm, problem instances with between 10 and 20 nodes are generated. The problem instances are generated similarly to what is done in [19]. The nodes are placed in a plane with the coordinates uniformly distributed between 0 and 100. The fixed costs $\left(c_{e}\right)$ are determined as the Euclidean distance. Rather than generating both capacity costs $\left(b_{e}\right)$ and the demands $\left(d_{i j}^{\prime}\right)$ and compute an average cost per ring per unit of demand to obtain $d_{i j}$ (as discussed in Section 3), we generate $d_{i j}$ only. The $d_{i j}$ values are generated as uniformly distributed between 0 and an upper bound $u$.

Selecting a proper value of $u$ is critical. If $u$ is selected too small, then the optimal solution is a single federal-ring including all nodes and no metro-rings. Using the same value of $u$ as in [19] proved sufficient. The upper bound $u$ used is given in the following equation.

$$
u \approx \frac{5}{\sqrt{|V|^{3}}}
$$

The value of $u$ arise by considering the tradeoff between total average demand and the shortest tour measured in fixed link costs for rings with $|V| / 2$ nodes. We refer to [19] for an in-depth explanation. The important observation is, that a tradeoff exists between the fixed link cost and the savings obtained from demands. As we shall see, the hop constraint (15) is in most, but not all cases binding; thus a tradeoff exists. The tests were run on a $1200 \mathrm{Mhz}$ SUN Fire 3800. We use CPLEX 9.0 to solve linear programming models.

For each of 10, 12,14, 16, 18 and 20 nodes, 10 different random instances are generated. We report results as averages over 10 instances. We vary the maximal number of nodes in the metro-rings, $m$ between 4 and $\min \{10,|V|-3\}$. In addition to this, we investigate networks with 25 and 36 nodes with $m$ equal to 5 and 6 respectively. It turns 
out, that since $|V| / m$ is integer for these networks, they are easier to solve than networks for which this is not the case. The results are given in Table 1 . The table shows the number of nodes, the maximum number of nodes in metro-rings, the number of branch-nodes, the total time spent in seconds and the percentage spent on the ring-partitioning problem and the ring-generation problem respectively. Finally the number of times that metrorings are generated (this includes cases where no metro-rings are actually found) and the number of metro-rings in the optimal solution are listed.

For all problem instances with up to 20 nodes, the branch-and-price algorithm terminates in at most 3 hours (average worst case is 73 minutes). Since the design of HRNs are considered strategic problems, the computational time is acceptable. As it can be seen, the bottleneck in the algorithm is the generation of rings which consistently takes more than $90 \%$ of the running time. The gradually increasing running time for increasing $|V|$ may both be attributed to increased running time for each ring-generation problem solved and to the increasing number of metro-rings which are generated (second last column). The number of branch-nodes is limited, making memory issues negligible. However, each branch requires generation of a substantial number of metro-rings, causing substantially higher running time.

Instances where $|V| / m$ is integer are easier than instances where this is not the case. This is due to the increased number of branch-nodes which is caused by an increased amount of fractional variables. Especially when $|V| / m=2$, the possibility of obtaining an integer solution without branching is high. The special case when all metro-rings and the federal-ring have the same number of nodes, i.e. $|V| / m=m$ and $|V| / m$ integer, is considered in $[14,15,16]$. Since $|V| / m$ is integer, as discussed above, such instances are easier to solve to optimality than instances where this is not the case. The last two rows in Table 1 gives results for instances with $|V|=25, m=5$ and $|V|=36, m=6$. The most difficult instances with 36 nodes are solved in less than 6 hours and on average over 10 instances in just above $1 \frac{1}{2}$ hour.

For networks with up to 20 nodes, in most cases, the optimal solution contains exactly the minimum number of metro-rings needed, given $m$. Only in 22 cases out of 380 test runs in total, one more than the minimum number of metro-rings needed is in the optimal solution. In Table 1, this is the reason why the last column contains fractions. This indicates that the demand values are sufficiently high to make the metrorings profitable and the hop constraint (15) thus binding. On the other hand, since some instances exists for which this is not the case, the demand values are not too high.

\subsection{Future research}

The approach described in this paper can handle instances of the modified HRN problem with up to 20 nodes. While we would like to solve larger problems, the discussion in Section 1 should make clear that the modified HRN problem constitutes a hard optimization problem. Furthermore, previous HRN approaches have either considered 
Table 1

Computational results. Averages over 10 instances.

\begin{tabular}{|c|c|c|c|c|c|c|c|}
\hline$|V|$ & $m$ & $\begin{array}{c}\text { \#Branch } \\
\text { Nodes }\end{array}$ & $\begin{array}{l}\text { Total Time } \\
\quad(\mathrm{sec} .)\end{array}$ & $\begin{array}{c}\text { Time } \\
\text { Part. }(\%)\end{array}$ & $\begin{array}{c}\text { Time } \\
\text { Gene. }(\%)\end{array}$ & $\begin{array}{l}\text { \#Rings } \\
\text { Gene. }\end{array}$ & $\begin{array}{c}\text { \#Metro } \\
\text { Rings }\end{array}$ \\
\hline 10 & 4 & 8.0 & 4.0 & 6.7 & 93.3 & 40.2 & 3.0 \\
\hline 10 & 5 & 1.0 & 2.5 & 4.6 & 95.4 & 18.8 & 2.0 \\
\hline 10 & 6 & 11.2 & 7.8 & 4.7 & 95.3 & 64.4 & 2.0 \\
\hline 10 & 7 & 7.2 & 4.9 & 5.7 & 94.3 & 42.1 & 2.0 \\
\hline 12 & 4 & 4.0 & 3.7 & 4.9 & 95.1 & 23.0 & 3.0 \\
\hline 12 & 5 & 13.0 & 16.4 & 3.9 & 96.1 & 83.2 & 3.0 \\
\hline 12 & 6 & 1.0 & 7.3 & 3.1 & 96.9 & 30.1 & 2.0 \\
\hline 12 & 7 & 15.0 & 24.5 & 3.5 & 96.5 & 105.5 & 2.0 \\
\hline 12 & 8 & 19.2 & 27.6 & 3.7 & 96.3 & 123.4 & 2.0 \\
\hline 12 & 9 & 9.8 & 14.1 & 3.6 & 96.4 & 65.4 & 2.0 \\
\hline 14 & 4 & 3.2 & 5.7 & 4.7 & 95.3 & 27.2 & 4.0 \\
\hline 14 & 5 & 6.4 & 18.5 & 2.6 & 97.4 & 51.1 & 3.0 \\
\hline 14 & 6 & 44.4 & 105.1 & 2.7 & 97.3 & 277.3 & 3.0 \\
\hline 14 & 7 & 1.0 & 29.8 & 1.4 & 98.6 & 46.8 & 2.0 \\
\hline 14 & 8 & 32.6 & 110.2 & 2.9 & 97.1 & 275.8 & 2.0 \\
\hline 14 & 9 & 50.6 & 118.5 & 3.2 & 96.8 & 327.5 & 2.0 \\
\hline 14 & 10 & 36.4 & 85.6 & 3.4 & 96.6 & 251.5 & 2.0 \\
\hline 16 & 4 & 5.8 & 11.9 & 4.2 & 95.8 & 39.6 & 4.4 \\
\hline 16 & 5 & 29.4 & 87.9 & 2.6 & 97.4 & 176.3 & 4.0 \\
\hline 16 & 6 & 34.4 & 158.4 & 2.1 & 97.9 & 251.1 & 3.0 \\
\hline 16 & 7 & 68.8 & 359.3 & 2.3 & 97.7 & 539.8 & 3.0 \\
\hline 16 & 8 & 1.8 & 84.2 & 0.9 & 99.1 & 68.7 & 2.0 \\
\hline 16 & 9 & 44.2 & 324.9 & 1.9 & 98.1 & 405.2 & 2.0 \\
\hline 16 & 10 & 60.6 & 383.0 & 2.5 & 97.5 & 570.7 & 2.0 \\
\hline 18 & 4 & 16.4 & 32.3 & 3.6 & 96.4 & 74.2 & 5.0 \\
\hline 18 & 5 & 2.6 & 32.4 & 1.8 & 98.2 & 40.3 & 4.0 \\
\hline 18 & 6 & 6.0 & 89.5 & 1.2 & 98.8 & 75.5 & 3.2 \\
\hline 18 & 7 & 33.4 & 446.5 & 1.3 & 98.7 & 325.6 & 3.0 \\
\hline 18 & 8 & 124.0 & 1183.7 & 2.1 & 97.9 & 1116.0 & 3.0 \\
\hline 18 & 9 & 1.0 & 217.3 & 0.6 & 99.4 & 89.9 & 2.0 \\
\hline 18 & 10 & 30.2 & 737.2 & 1.3 & 98.7 & 440.2 & 2.0 \\
\hline 20 & 4 & 6.8 & 27.0 & 3.9 & 96.1 & 50.6 & 5.7 \\
\hline 20 & 5 & 8.6 & 91.8 & 1.5 & 98.5 & 71.7 & 4.7 \\
\hline 20 & 6 & 24.2 & 356.4 & 1.1 & 98.9 & 190.3 & 4.0 \\
\hline 20 & 7 & 12.8 & 407.0 & 0.7 & 99.3 & 143.4 & 3.2 \\
\hline 20 & 8 & 53.4 & 1854.6 & 0.9 & 99.1 & 659.6 & 3.0 \\
\hline 20 & 9 & 179.8 & 4344.2 & 1.4 & 98.6 & 2026.2 & 3.0 \\
\hline 20 & 10 & 1.0 & 688.0 & 0.3 & 99.7 & 117.8 & 2.0 \\
\hline 25 & 5 & 11.2 & 302.0 & 1.0 & 99.0 & 110.8 & 5.9 \\
\hline 36 & 6 & 21.0 & 5457.8 & 0.4 & 99.6 & 245.9 & 7.0 \\
\hline
\end{tabular}


heuristics, with no performance guarantees, or enumerative schemes which can handle less than 12 nodes. In order to be able to handle larger instances in reasonable time, it is paramount to reduce the time spent on ring-generation. Note that for each branchnode in the branch-and-bound algorithm, at least one ring-generation problem has to be solved to optimality (the one giving no rings) to ensure that the value obtained when solving the ring-partitioning problem is indeed a bound. Thus it is inevitable that the ring-generation problem has to be solved to optimality at least as many times as there are branch-nodes. The remaining number of times that rings are generated heuristics could be used, rings could be pre-generated and several rings could be generated each time. These techniques could probably increase the size of the modified HRN problems which can be handled. In this paper we have further considered the most abstract and general formulation of the problem. An obvious practical improvement could be to limit the links allowed in the network to the e.g. $k$ nearest neighbours for each node. Another approach to reduce the problem hardness is the use of a so-called compatibility graph which disallow certain pairs of nodes to be in the same metro-network is suggested in [11].

As mentioned in Section 3, the optimal solution of the modified HRN problem may not be optimal in the original HRN problem. This is mainly for two reasons: The metro-rings and the federal-ring is designed in separate (thus non-optimal) stages and secondly, the modification assumes the average cost per ring per unit of demand of rings are the same. It seems possible but nontrivial to include the federal-ring design in the branch-and-price algorithm, but it seems more difficult to solve the problem with the cost per ring per unit of demand. However, one initial approach to take is to investigate how much the optimal solution for the modified HRN problem deviates from the optimal solution to the original HRN problem. This could be done either by investigating very small instances for which optimal solutions can be found or by finding a lower bound on the original problem cost.

Also it would be interesting to allow bidirectional instead of unidirectional selfhealing rings. One possibility is to use the same problem modification, and thus approximate the bidirectional rings with unidirectional rings. However, in that case the traffic approximation becomes even more unreliable.

Note that a capacity constraint can be added to the ring-generation problem, thus dealing with ring capacity for the metro-rings. If capacities are available in modular sizes for varying capacity costs, an idea is to generate rings for each of the available capacities and corresponding capacity cost. This will probably not work very well, essentially because the capacity cost per ring per unit of demand is different for rings and thus $B$ is not a good estimate for at least some rings. It may be possible to get reasonable results, however, by using different estimates of $B$ for the various capacities. Assume a low capacity ring has a high per unit capacity cost. In that case, intuitively a high $B$ should be used for low capacity rings, since this will correspond to a low overall saving accounting for the more expensive per unit capacity, see equation (4). 


\section{Conclusion}

In this paper we have considered the problem of designing HRNs. A problem modification has been presented which has previously been used to build heuristics for designing HRNs. A branch-and-price algorithm is described, implemented and tested. For the modified problem this algorithm finds provably optimal solutions to networks with up to 20 nodes in less than 3 hours. For problems with special structure, the algorithm finds provably optimal solutions with up to 36 nodes in less than 6 hours. The computational time depends heavily on the instances considered, and in particular it is possible to design considerably larger networks if the maximum number of nodes in metro-rings are small and/or if the number of nodes in the network is divisible with the maximum number of nodes in metro-rings. Algorithmic improvements which could speed up the algorithm have been suggested and we also suggest an investigation of how much the optimal solution to the modified problem deviates from the solution to the original problem. In particular this investigation is important if bidirectional self healing rings are considered.

\section{References}

[1] K. Altinkemer, Topological design of ring networks, Computers \& Operations Research 21(4) (1994) 421-431.

[2] C. Barnhart, E.L. Johnson, G.L. Nemhauser, M.W.P. Savelsbergh and P.H. Vance, Branch-and-price: Column generation for solving huge integer programs, Operations Research 46(3) (1998) 316-329.

[3] M. Fischetti, J.J. Salazar Gonzalez and P. Toth, A branch-and-cut algorithm for the symmetric generalized traveling salesman problem, Operations Research 45(3) (1997) 378-394.

[4] T. Flanagan, Fiber network survivability, IEEE Communications Magazine 28(6) (1990) 46-53.

[5] B. Fortz, P. Soriano and C. Wynants, A tabu search algorithm for self-healing ring network design, European Journal of Operational Research 151(2) (2003) 280-295.

[6] M. Gawande, J.G. Klincewicz and H. Luss, Design of SONET/SDH ring assignment with capacity constraints, Advances in Performance Analysis 2 (2000) 159-217.

[7] O. Goldschmidt, A. Laugier and E.V. Olinick, SONET/SDH ring assignment with capacity constraints, Discrete Applied Mathematics 129(1) (2003) 99-128.

[8] D. Kang, K. Lee, S. Park, K. Park and S.-B. Kim, Design of local networks using USHRs, Telecommunication Systems 14(4) (2000) 197-217.

[9] J.G. Klincewicz, Hub location in backbone/tributary network design: A review, Location Science 6 (1998) 307-333.

[10] V. Mak and T. Thomadsen, Facets for the Cardinality Constrained Quadratic Knapsack Problem and the Quadratic Selective Travelling Salesman Problem, IMM-Technical Report-2004-19 (2004).

[11] K. Park, K. Lee, S. Park and H. Lee, Telecommunication node clustering with node compatibility and network survivability requirements, Management Science 46(3) (2000) 363-374.

[12] A. Proestaki and M.C. Sinclair, Design and dimensioning of dual-homing hierarchical multi-ring networks, IEE Proceedings-Communications 147(2) (2000) 96-104.

[13] D.M. Ryan and B. Foster, An integer programming approach to scheduling, in: Computer Scheduling of Public Transport. Urban Passenger Vehicle and Crew Scheduling. Proceedings of an International Workshop (1981) pp. 269-280.

[14] J. Shi and J.P. Fonseka, Dimensioning of self-healing rings and their interconnections, in: Global Telecommunications Conference, 1993, Including a Communications Theory Mini-Conference. 
Technical Program Conference Record, IEEE in Houston. GLOBECOM '93., IEEE Vol. 3 (1993) pp. 1579-1583.

[15] J. Shi and J.P. Fonseka, Design of hierarchical self-healing ring networks, in: Communications, 1994. ICC '94, SUPERCOMM/ICC '94, Conference Record, 'Serving Humanity Through Communications.' IEEE International Conference on (1994) Vol. 1, pp. 478-482.

[16] J. Shi and J.P. Fonseka, Hierarchical self-healing rings, IEEE/ACM Transactions on Networking (1995) 690-697.

[17] J. Shi and J.P. Fonseka, Interconnection of self-healing rings, in: 1995 IEEE International Conference on Communications. Converging Technologies for Tomorrow's Applications, ICC '96 (1996) Vol. 3 , pp. $1563-1567$.

[18] J. Shi and J.P. Fonseka, Analysis and design of survivable telecommunications networks, IEE Proceedings-Communications 144(5) (1997) 322-330.

[19] T. Thomadsen and T. Stidsen, The quadratic selective travelling salesman problem, IMM-Technical Report-2003-17 (2003).

[20] F. Vanderbeck and L.A. Wolsey, An exact algorithm for IP column generation, Operations Research Letters 19 (1996) 151-159. 